\title{
Measurement of female genital mutilation/cutting status: Perspectives from healthcare providers, policymakers, programme implementers, and researchers
}

Dennis Matanda

Population Council

Follow this and additional works at: https://knowledgecommons.popcouncil.org/departments_sbsr-rh

Part of the Demography, Population, and Ecology Commons, Family, Life Course, and Society Commons, International Public Health Commons, and the Sociology of Culture Commons How does access to this work benefit you? Let us know!

\section{Recommended Citation}

Matanda, Dennis. 2020. "Measurement of female genital mutilation/cutting status: Perspectives from healthcare providers, policymakers, programme implementers, and researchers," Evidence to End FGM/C: Research to Help Girls and Women Thrive. New York: Population Council. 


\section{Evidence to End FGM/C}

Research to Help Girls and Women Thrive

\section{MEASUREMENT OF FEMALE GENITAL MUTILATION/CUTTING STATUS PERSPECTIVES FROM HEALTHCARE PROVIDERS, POLICYMAKERS, PROGRAMME IMPLEMENTERS, AND RESEARCHERS}

April 2020 


\section{MEASUREMENT OF FEMALE GENITAL MUTILATION/CUTTING STATUS}

PERSPECTIVES FROM HEALTHCARE PROVIDERS, POLICYMAKERS, PROGRAMME IMPLEMENTERS, AND RESEARCHERS

DENNIS MATANDA POPULATION COUNCIL 
Evidence to End FGM/C: Research to Help Girls and Women Thrive generates evidence to inform and influence investments, policies, and programmes for ending female genital mutilation/cutting in different contexts. Evidence to End FGM/C is led by the Population Council, Nairobi in partnership with the Africa Coordinating Centre for the Abandonment of Female Genital Mutilation/Cutting (ACCAF), Kenya; the Global Research and Advocacy Group (GRAG), Senegal; Population Council, Nigeria; Population Council, Egypt; Population Council, Ethiopia; MannionDaniels, Ltd. (MD); Population Reference Bureau (PRB); University of California, San Diego (Dr. Gerry Mackie); and University of Washington, Seattle (Prof. Bettina Shell-Duncan).

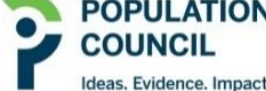

Ideas. Evidence. Impact.
The Population Council confronts critical health and development issues-from stopping the spread of HIV to improving reproductive health and ensuring that young people lead full and productive lives. Through biomedical, social science, and public health research in 50 countries, we work with our partners to deliver solutions that lead to more effective policies, programmes, and technologies that improve lives around the world. Established in 1952 and headquartered in New York, the Council is a nongovernmental, nonprofit organisation governed by an international board of trustees. www.popcouncil.org

Suggested Citation: Matanda, Dennis. 2020. "Measurement of Female Genital Mutilation/Cutting Status: Perspectives from Healthcare Providers, Policymakers, Programme Implementers, and Researchers." Evidence to End FGM/C: Research to Help Girls and Women Thrive. New York: Population Council.

This is a working paper and represents research in progress. This paper represents the opinions of the author and is the product of professional research. This paper has not been peer reviewed, and this version may be updated with additional analyses in subsequent publications. Contact: dmatanda@popcouncil.org

Please address any inquiries about the Evidence to End FGM/C programme consortium to:

Dr Jacinta Muteshi, Project Director, jmuteshi@popcouncil.org

Funded by:

This document is output from a programme funded by UK Aid from the UK government for the benefit of developing countries. However, the views expressed and information contained in it are not necessarily those of, or endorsed by the UK government, which can accept no responsibility for such views or information or for any reliance placed on them. 


\section{Table of Contents}

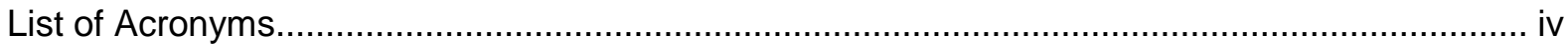

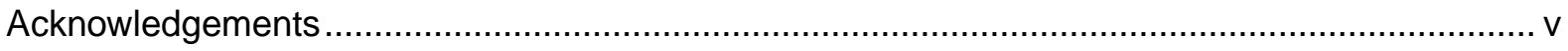

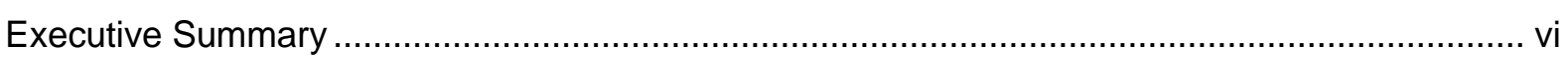

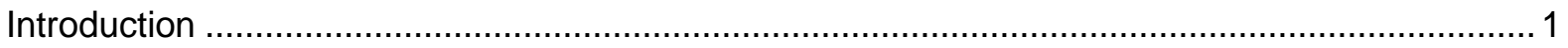

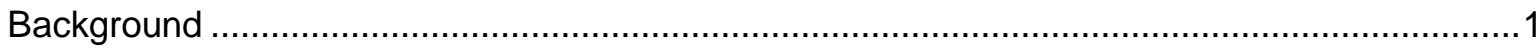

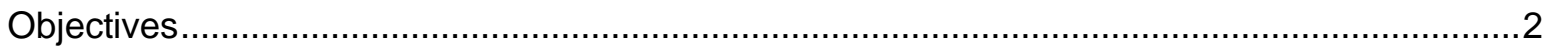

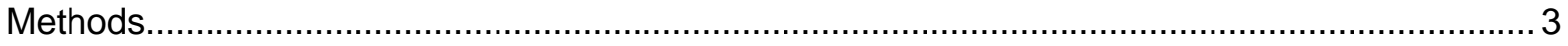

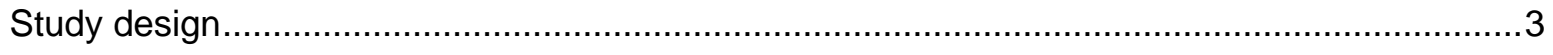

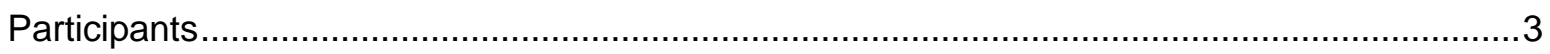

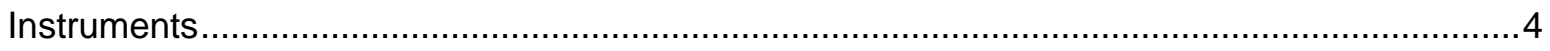

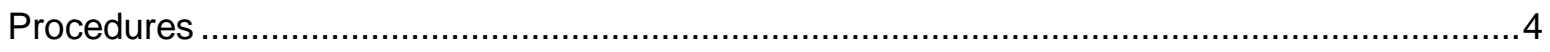

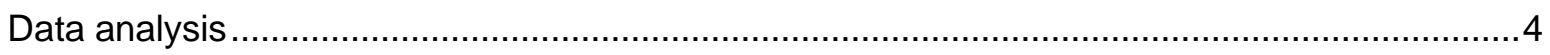

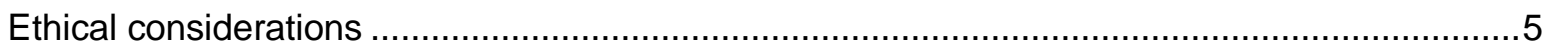

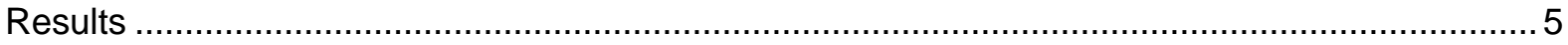

Advantages and challenges of having healthcare providers physically examine and report on

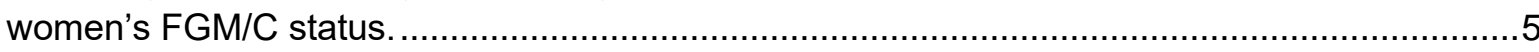

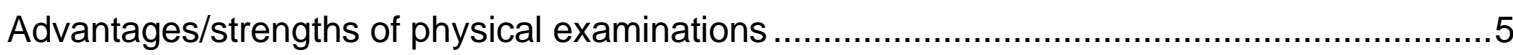

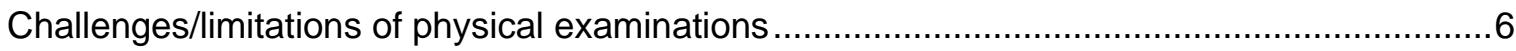

Feasibility of conducting physical examinations on women/girls to collect data on FGM/C.....9

Advantages and challenges of women and girls self-reporting on FGM/C ............................10

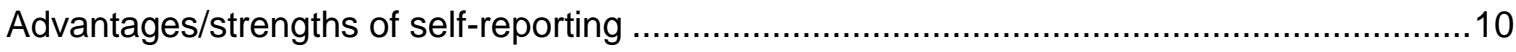

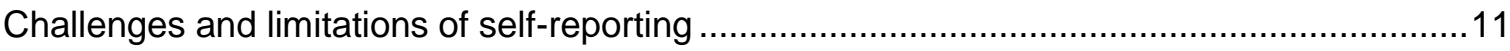

Validity of responses from parents/guardians reporting their daughter's FGM/C status...........12

Preferred approaches in measuring FGM/C prevalence .....................................................14

Views about WHO classification of the different types of FGM/C .........................................15

Feasibility of using images in identifying and classifying various types of FGM/C.................16

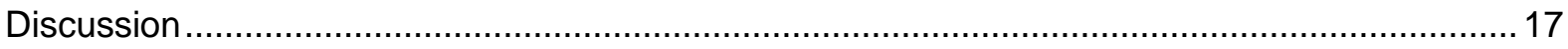

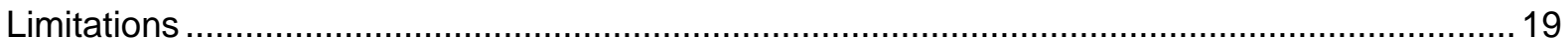

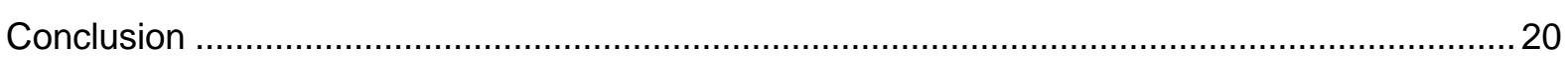

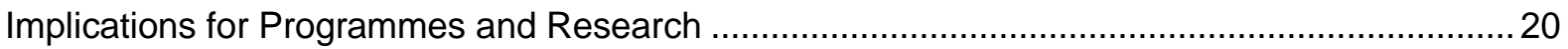

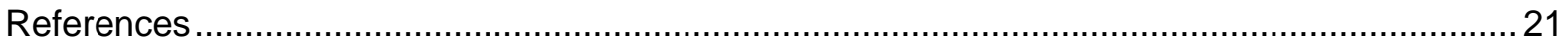




\section{List of Acronyms}

$\begin{array}{ll}\text { ANC } & \text { Antenatal Care } \\ \text { DfID } & \text { Department for International Development } \\ \text { DHS } & \text { Demographic and Health Survey } \\ \text { DRC } & \text { Democratic Republic of Congo } \\ \text { FGC } & \text { Female Genital Cutting } \\ \text { FGM } & \text { Female Genital Mutilation } \\ \text { FGM/C } & \text { Female Genital Mutilation/Cutting } \\ \text { UK } & \text { United Kingdom } \\ \text { USA } & \text { United States of America } \\ \text { MCH } & \text { Maternal and Child Health } \\ \text { WHO } & \text { World Health Organisation }\end{array}$




\section{Acknowledgements}

We are grateful to healthcare providers, policymakers, programme implementers, and researchers who took their valuable time to respond to the online survey. We acknowledge input from Otibho Obianwu who participated in the design of the study questionnaire. Chantalle Okondo played a key role in sending out the survey to potential participants and translating transcripts from French to English. Many thanks to Stanley Yoder, Caroline Kabiru, and Jacinta Muteshi for reviewing earlier drafts of this report and providing critical feedback. Additional technical and editorial support was provided by Michelle Hindin and the Population Council Publication Team.

This work was funded by UK Aid and the UK Government through the DfID-funded project "Evidence to End FGM/C: Research to Help Girls and Women Thrive" coordinated by the Population Council. 


\section{Executive Summary}

\section{Background}

Among practising communities, female genital mutilation/cutting (FGM/C) is a highly sensitive and emotionally charged issue, and open discussion is often considered a taboo. Not only may it be difficult to motivate people to discuss the topic, but the validity of their responses may be questionable. These factors make research on $\mathrm{FGM} / \mathrm{C}$ challenging and hence the need for careful consideration of research methodologies. While physical exam/confirmation could be a gold standard methodology when conducting research on $\mathrm{FGM} / \mathrm{C}$, it is rarely done. It is also difficult to get population prevalence data with physical exams. Consequently, most of the data on FGM/C comes from self-reported survey responses. Even though studies have been conducted to assess the validity and reliability of some of these approaches in collecting FGM/C data, the debate on the validity and reliability of $\mathrm{FGM} / \mathrm{C}$ data persists. There is a need for proper documentation of strengths and limitations of the various data-collection methodologies to assist in generating reliable data for programmatic efforts towards FGM/C abandonment. We therefore undertook a study to highlight methodological opportunities and challenges in measuring the prevalence, drivers, and dynamics of FGM/C. The specific objectives were to:

- Assess the advantages and challenges of having healthcare providers physically examine and report on women's FGM/C status;

- Understand the advantages and challenges of self-reporting as a method of data collection for FGM/C;

- Explore the validity of responses from parents or guardians reporting their daughter's FGM/C status;

- Identify the preferred approaches in measuring FGM/C prevalence; and

- Assess participants' views about the WHO classification of the different types of FGM/C.

\section{Methods}

We conducted a cross-sectional qualitative study. Data were collected using an online questionnaire with open-ended questions. Study participants included professionals working in the area of $\mathrm{FGM} / \mathrm{C}$ either as healthcare providers, policymakers, programme implementers or researchers. A total of 51 professionals across the globe voluntarily agreed to participate in the study. NVivo version 11 software was used to conduct data analysis. The study team used an inductive analysis approach to review text data and identify themes and patterns.

\section{Results}

Physical examinations were reported to provide opportunities for identification and treatment of health complications related to FGM/C and a platform for sensitisation and prevention of $\mathrm{FGM} / \mathrm{C}$. This data-collection approach also had its challenges such as the high possibility that $\mathrm{FGM} / \mathrm{C}$ survivors would avoid routine visits to health facilities especially in contexts where healthcare providers are required to report cases to authorities, lack protocol on how physical examinations are to be conducted, capability of the healthcare system to document and report FGM/C cases is limited, and healthcare providers demonstrate inadequate knowledge about FGM/C.

Participants highlighted several benefits of self-reported FGM/C status. Self-reporting was considered less intrusive, makes it possible to reach a larger study population and captures wideranging information within a short period of time, and was effective in documenting forms of $\mathrm{FGM} / \mathrm{C}$ 
that cannot be physically seen. Nonetheless, the approach was equally limited with challenges such as difficulties in reconciling interviewer versus interviewee definition of $F G M / C$ and the description of the various types of FGM/C, respondents having experienced FGM/C at younger ages and hence finding it difficult to remember the events in detail, and under-reporting because of the legal status. The validity of parents' or guardians' reports of daughters' FGM/C status is affected by contextual issues such as existence of laws prohibiting FGM/C, whether FGM/C was considered a norm in the respective study population, and the level of ongoing anti-FGM/C interventions in the community.

Whereas the WHO classification of the different types of FGM/C has been an important step in aiding measurement of $\mathrm{FGM} / \mathrm{C}$, professionals working on $\mathrm{FGM} / \mathrm{C}$ reported several challenges while using these classifications. Classification of FGM/C Type IV was specifically contentious regarding its relevance and application. Inadequate understanding of the WHO classifications by healthcare providers was a significant barrier towards their effective use. Given the noted challenges in identifying and classifying various forms of $F G M / C$, the use of images/pictorial presentations of FGM/C presents an opportunity to improve accuracy in the identification and classification process.

\section{Discussion}

The strengths and challenges of physical examinations, self-reporting, and the use of parents' or guardians' reports call for caution when interpreting data on FGM/C generated through these approaches. Specifically, contextual factors such as existence of anti-FGM/C laws, ongoing anti$\mathrm{FGM} / \mathrm{C}$ interventions in the community, and whether $\mathrm{FGM} / \mathrm{C}$ is a norm in the community need to be taken into account. Various studies have highlighted some of these strengths and limitations regarding approaches used in collecting FGM/C data. What our study adds is a much-needed resource that documents key $\mathrm{FGM} / \mathrm{C}$-related measurement considerations that programme implementers and researchers need to be cognisant of.

Study findings on the WHO classifications of the different types of FGM/C highlighted various challenges in their implementation. Whereas the WHO classifications of the different types of FGM/C has been an important milestone in the description and comparison of the practise, the different forms of $\mathrm{FGM} / \mathrm{C}$ are complex. Precise anatomical classification relies on examination of the genitalia by an experienced examiner. Possibilities also exist for self-report using line drawings of $\mathrm{FGM} / \mathrm{C}$ type for self-assessments.

\section{Implications for programmes and research}

- Programme implementers and researchers utilising various approaches in collecting FGM/C data need to be conscious of the specific strengths and limitations of each approach. For monitoring prevalence, data generated from women's self-reports or parents' or guardians' reports on their daughters' FGM/C status have strengths and weaknesses especially for tracking abandonment. For treatment and care, caution should be observed when utilising data generated through physical exams and self-reports. Importantly, this study has documented opportunities and challenges regarding collection of data on $\mathrm{FGM} / \mathrm{C}$, and therefore provides a unique resource for programme implementers, researchers, and other stakeholders interested in the data-collection discourse on sensitive issues such as FGM/C.

- There is a need for research on the relevance and applicability of the WHO classifications of the different types of FGM/C. More research should also be conducted to provide clear guidelines on how images/pictorial presentations of $\mathrm{FGM} / \mathrm{C}$ can be used in the identification, classification, and management of FGM/C cases. 


\section{Introduction}

\section{Background}

The lack of high-quality data on the practice of female genital mutilation/cutting (FGM/C) and other harmful practices limits the design and implementation of effective interventions. This is because the practice occurs in private with effects not externally visible and the topic sensitive; therefore, people may be inclined to hide FGM/C status. For instance, in situations where FGM/C is illegal, people may under-report the practice or their opinion on its continuation because of the fear for prosecution (Askew 2005). Alternatively, in contexts where the prevalence is high and the practice considered a norm, people may overstate its prevalence and their preference for its continuation due to the social pressure to conform (Huntington et al. 1996; Morison et al. 2001; Snow et al. 2002). Consequently, the possibility that people can under-report or overstate the prevalence introduces errors in estimating the true prevalence of $\mathrm{FGM} / \mathrm{C}$ and assessing possible correlates of the practice (Gibson et al. 2018).

Many studies on FGM/C, especially those that utilise population survey data, have relied on selfreports either from a woman or a parent or guardian reporting on behalf of the daughter (Elmusharaf, Elhadi, and Almroth 2006; Gibson et al. 2018). Studies utilising physical examination as an approach in data collection on $\mathrm{FGM} / \mathrm{C}$ are rare due to the intrusiveness of the approach and the associated costs. An example of a large-scale study that used physical examination to collect data on FGM/C was conducted by the World Health Organisation (WHO 2006). The study involved six African countries and was conducted at 28 obstetric centres where women who presented for singleton delivery were interviewed to obtain information about their personal characteristics and obstetric and medical histories. Study participants also underwent antepartum examination of the external genitalia to determine their FGM/C status, and if they had been cut, to ascertain the type of $\mathrm{FGM} / \mathrm{C}$ (WHO 2006). Even though considered the gold standard approach in data collection on FGM/C (Elmusharaf, Elhadi, and Almroth 2006), findings from studies that have investigated the validity of physical examination have shown some discordance (Elmusharaf, Elhadi, and Almroth 2006; Klouman, Manongi, and Klepp 2005). Published data indicate that women and clinicians may both incorrectly report women's FGM/C status. Further, self-reporting by parents or guardians reporting on their daughters' status may present certain constraints. These methodological challenges have an impact on the accurate measurement of changes in the practice (Adinma 1997; Morison et al. 2001; Odujinrin, Akitoye, and Oyediran 1989; Toubia 1994).

Research on FGM/C can be extremely challenging for a number of reasons. Among practising communities, the topic of $\mathrm{FGM} / \mathrm{C}$ is a highly sensitive and emotionally charged issue, and open discussion with outsiders is often considered a taboo. Not only may it be difficult to motivate people to discuss the topic (individually or in small groups), but they may intentionally misreport. There is a need for accurate approaches for measurement of FGM/C, as this is critical to the success of the research agenda (Jackson et al., 2003). Quality data on FGM/C are needed to ensure that evaluation studies of FGM/C interventions, assessment of determinants of $F G M / C$, and estimates of prevalence and trends are accurate.

Another important area in measurement of $\mathrm{FGM} / \mathrm{C}$ relates to the definition and subsequent classification of the different types of $\mathrm{FGM} / \mathrm{C}$ as provided by the World Health Organisation (WHO) (OHCHR et al. 2008; WHO 1998). There is substantial variability in the types of FGM/C performed across countries and cultures. Against this background, the WHO in 1995 convened a technical working group to develop a standardised classification of FGM/C (Creighton and Hodes 2016; WHO 1998). This classification was to enabled more accurate description and comparison of the 
different types of FGM/C practised globally. The technical working group classified FGM/C into four groups based on the extent and amount of genital tissue removed (WHO 1998). The classification was further reviewed and updated in 2008. The most recent classification of FGM/C practises by WHO comprises: Type I (clitoridectomy), partial or total excision of the clitoris or its prepuce or both; Type II (excision), partial or total excision of the clitoris in addition to labia minora or labia majora or both; Type III (infibulation), excision of part of the external genitalia and adhesion of the labia minora or majora or both to enclose the vulva; and Type IV brings together all other unclassified harmful procedures to the female genitalia including pricking, piercing, incising, scraping, and cauterisation (OHCHR et al. 2008).

Over the years, experience with the use of the 1998 WHO classification of FGM/C raised some ambiguities, which were addressed in a revision issued in 2008 that incorporated some modifications to accommodate concerns and shortcomings of the 1998 classifications, while maintaining the four types (OHCHR et al. 2008; WHO 1998). Important to note is that the 2008 review concluded that the available evidence by then was insufficient to warrant a new classification. Therefore, only slight modifications were made in the wording of the typology, and sub-divisions created to accommodate increased variety of $F G M / C$ procedures. Over a decade has now passed since the adoption of the $2008 \mathrm{WHO}$ classification of FGM/C. It is imperative to find out views and experiences of stakeholders working in the area of FGM/C on how these classifications have been applied over time. Misclassification of the different types of FGM/C has implications for clinical interventions that use these classifications for care and management of FGM/C-related complications. Misclassification also has research implications as it affects the accuracy and reliability of $\mathrm{FGM} / \mathrm{C}$ indicators.

It is against this background that the Population Council, informed by its collective experience implementing FGM/C research, undertook this study to highlight methodological opportunities and challenges in measuring the prevalence, drivers, and dynamics of FGM/C. This study was a followup to discussions held on 20 May 2019 in Brussels during the Population Council-led satellite workshop of the Third G3 International Expert Meeting on FGM/C entitled FGM/C Prevalence Studies: Direct Observation versus Self-Reporting (Direct and Indirect) in Source and Migration Countries. The workshop explored the following questions:

1) What is the best way to measure the prevalence of $F G M / C$ ?

2) What are the ethical concerns and feasibility of direct observation measures?

3) What can we do for population-level estimates? - what are the challenges associated with Type IV when conducting population-level estimates?

\section{Objectives}

The aim of the study was to gather data from professionals working in the area of FGM/C about their views and experiences in the measurement of FGM/C prevalence. Specifically, the study had the following objectives:

1) To assess the advantages and challenges of having healthcare providers physically examine and report on women's FGM/C status;

2) To understand the advantages and challenges of self-reporting as a method of data collection for FGM/C;

3) To explore the validity of responses from parents or guardians reporting their daughter's FGM/C status;

4) To identify the preferred approaches in measuring FGM/C prevalence; and 
5) To assess participants' views about the WHO classification of the different types of FGM/C.

\section{Methods}

\section{Study design}

We conducted a cross-sectional qualitative study. Data were collected using an online questionnaire with open-ended questions. Participants included professionals working in the area of FGM/C either as healthcare providers, policymakers, programme implementers, or researchers.

\section{Participants}

The questionnaire was sent out to an e-mail list of attendees of the Third G3 International Expert Meeting on FGM/C held in Brussels, Belgium. We also sent out the survey to other possible participants whose contacts were available but did not attend the expert meeting. Figure 1 summarises the number of study participants and their countries of residence. A total of 51 professionals agreed to participate in the study and filled out the online questionnaire. The largest proportion of participants were from Nigeria (15) followed by the United States (9) and Belgium (5).

\section{Figure 1. Number of participants and country of residence}

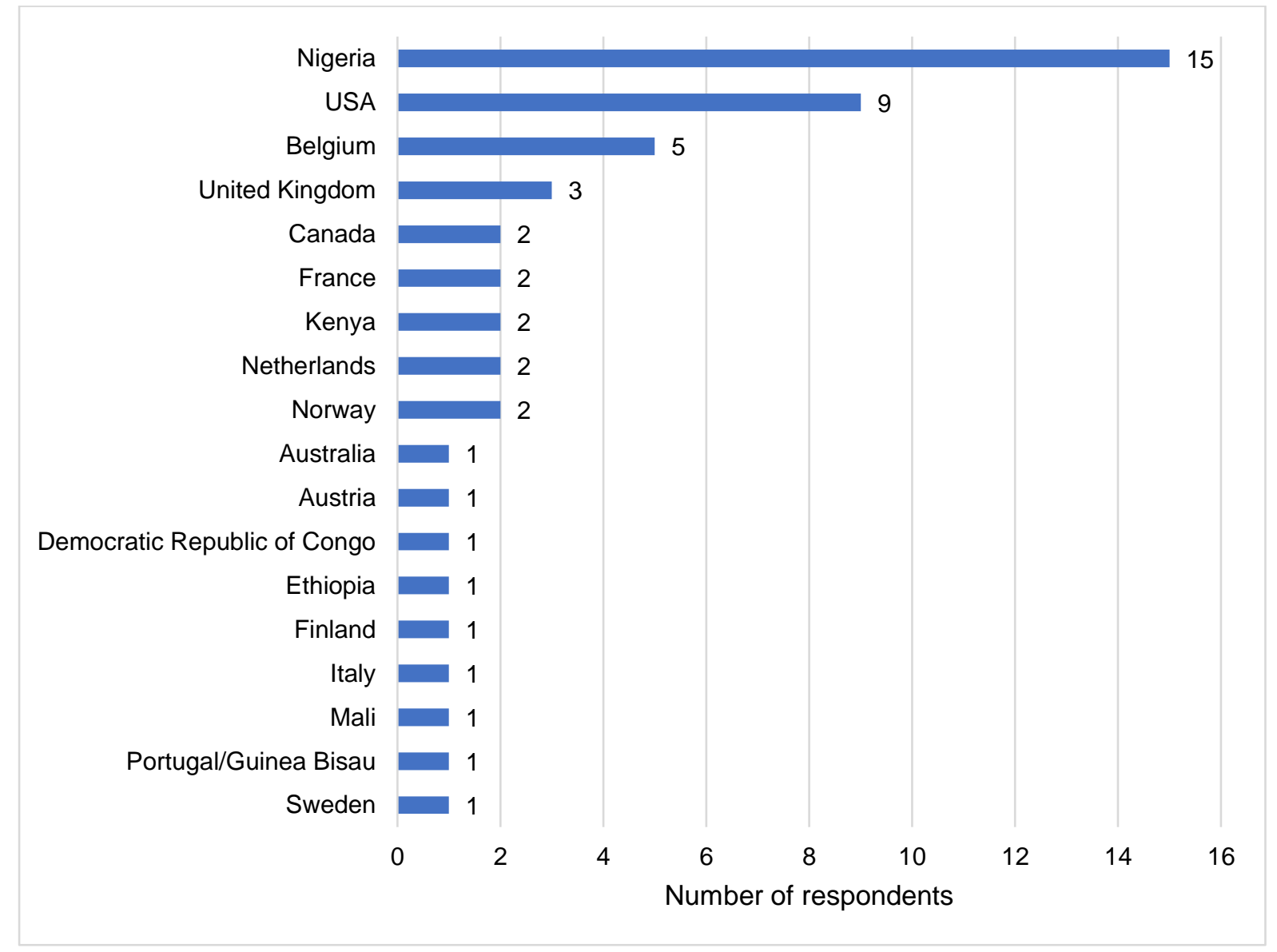

Figure 2 shows the proportion of participants by their main area of work with regard to FGM/C. The largest category of participants $(n=17)$ were working in research $(33 \%), 13$ participants were working in the healthcare sector as providers $(25 \%), 11$ were implementing programmes on $\mathrm{FGM} / \mathrm{C}(22 \%)$, eight were working in the Other category-they did not exclusively identify 
themselves as working either in healthcare, policy, programme implementation or research $(16 \%)$-while two of the participants were working in the policy sector $(4 \%)$.

Figure 2. Participants' main area of work related to FGM/C $(n=51)$

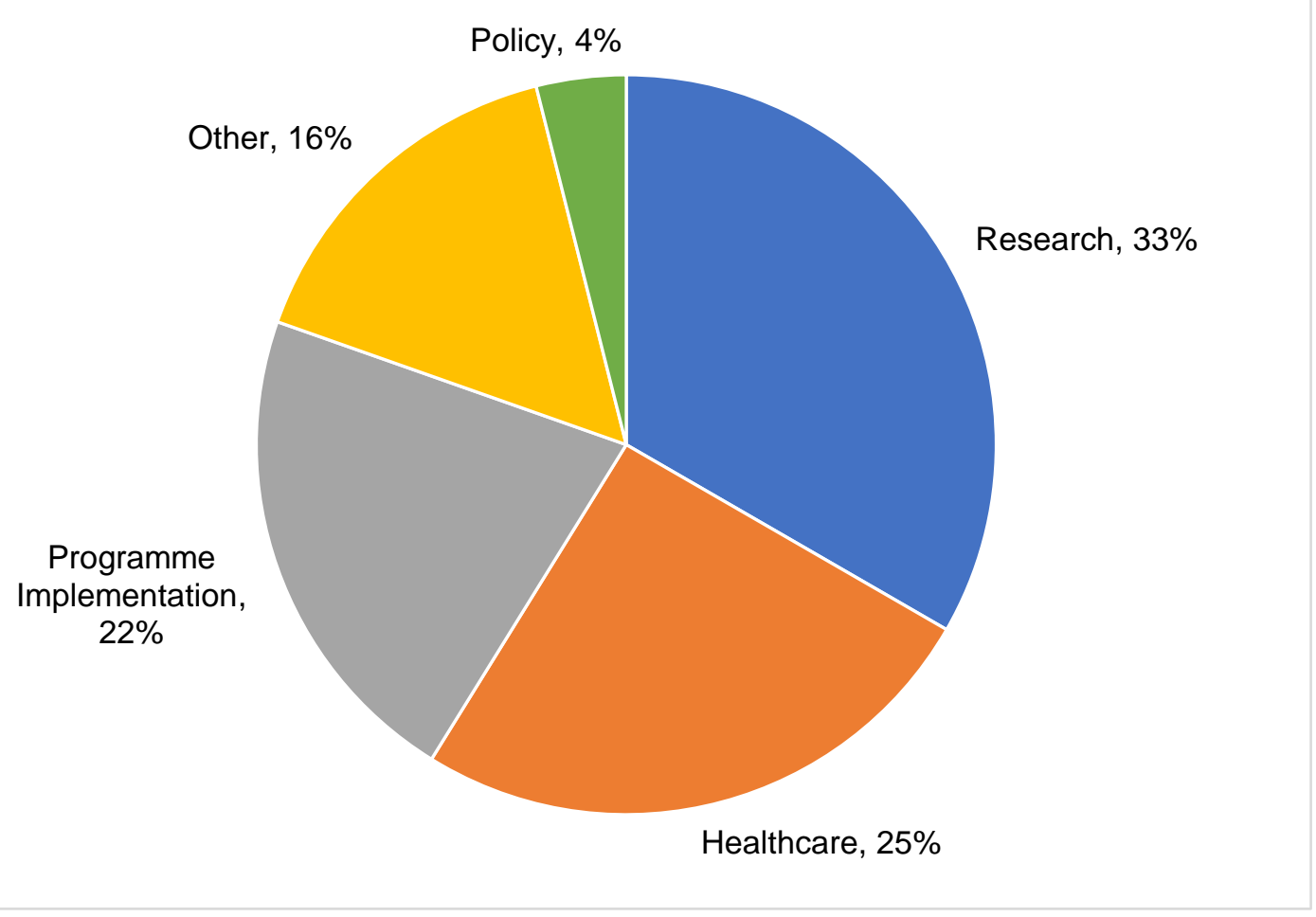

\section{Instruments}

The online questionnaire was comprised of open-ended questions. The development of the online questionnaire was informed by discussions during the satellite workshop in Belgium. The tool was reviewed internally by Population Council colleagues and updated before the final questionnaire was sent out to study participants.

\section{Procedures}

The study team wrote to the organising committee of the Third G3 International Expert Meeting on FGM/C held in May 2019 in Brussels, requesting a list of email addresses of attendees. We then used this list to reach out to possible study participants. The study team also reached out to other professionals who did not attend the meeting but whose email addresses were available. We used Google Forms-a free online platform - to create the survey and collect participants' responses. Data collection took place between June 19, 2019 and July 10, 2019.

\section{Data analysis}

Participants' responses were copied into Microsoft Word and exported to NVivo version 11 software for analysis. Responses in French (8) were directly translated into English. The study team used an inductive analysis approach to review text data, identify themes and patterns and construct typologies. Codes corresponding to themes and constructs were entered into the NVivo database and used to organise data and conduct refined analysis. 


\section{Ethical considerations}

We requested consent from participants before taking part in the survey. Participants were informed that their participation was voluntary, and their responses would be anonymous and used solely for research purposes.

\section{Results}

In this section we first highlight the reported advantages and challenges of having healthcare providers physically examine and report on women's FGM/C status. We then summarise the advantages and challenges of self-reporting as a method of data collection, the validity of responses from parents or guardians reporting on their daughter's FGM/C status, and preferred approaches in measuring FGM/C prevalence. Finally, we present participants' views about the WHO classification of the different types of FGM/C.

\section{Advantages and challenges of having healthcare providers physically examine and report on women's FGM/C status}

Physical examination of the female genitalia has been touted as the gold standard for assessing FGM/C status (Elmusharaf, Elhadi, and Almroth 2006). Nonetheless, it has been noted that the approach has its limitations (Klouman, Manongi, and Klepp 2005). We asked participants to share their experiences and thoughts on the strengths and limitations of conducting clinical exams to collect data on FGM/C.

\section{Advantages/strengths of physical examinations}

As illustrated in the following quotes, participants noted that physical examination offered an opportunity to identify and treat health complications related to FGM/C. Early detection of FGM/C was considered important as it aids prevention of chronic complications that are difficult and expensive to manage later in life. Physical examination also makes it possible to refer those with FGM/C-related complications for specialised treatment, but as noted by one respondent, the examiners may not assess properly.

"It will aid diagnosis, treatment and determining when and where to refer patients who need additional support and care. It will also aid in the identification of new cases of FGM."

Programme Implementer, Nigeria

"A well-documented physical exam allows for adequate post-exam treatment (deinfibulation, pain, urinary concerns, childbirth concerns, etc). Most examiners don't know how to do a proper assessment, and thus opportunity for optimising care is lost. A good examination would also allow referral for clitoroplasty."

Researcher and Healthcare Provider, Australia

"In the frame of physical examinations, the healthcare provider can screen for any problems that the woman may face, both physically and in terms of her well-being. They can answer questions. It may be important for the woman that the healthcare provider acknowledges the FGM/C that she has and makes it possible for her to speak about it. The healthcare provider can give information and refer to other specialists if needed, including a gynaecologist, a sexologist, or a psychologist."

$$
\text { Programme Implementer, Belgium }
$$

The second advantage of physical examination was the consideration that information gathered through clinical exams was more reliable. Participants noted that physical examination enables the examiner to observe and confirm whether the woman has been cut or not and also document more detailed information about the different types of FGM/C. Observation also reduced errors in 
situations where women/girls may not remember the experience of being cut, but there are likely many opportunities in their lives to know whether they are cut or not.

"The information will be more reliable when the genital area is examined, as women don't always remember whether the FGM has been performed on them and they don't necessarily recognise whether their genitalia is normal or cut."

Researcher, Finland

"The healthcare provider with the help of guides can be able to determine the type of cuts that the women or girls have undergone. It can also help address recall bias for example girls are being cut at younger ages, perhaps some may have forgotten they underwent the procedure or maybe they are not sure what type of cut they received."

Researcher, Kenya

Physical examination was seen to offer an opportunity for sensitisation and prevention of FGM/C as well as an opportunity to address broader reproductive health concerns. Participants noted that physical examination involves interaction with the woman/girl and therefore healthcare providers can take advantage of this opportunity to discuss issues around FGM/C and pass messages that can help in preventing $\mathrm{FGM} / \mathrm{C}$ for the uncut girls and reinfibulation for those already cut. This can also serve as an opportunity to address other reproductive health issues such as other forms of sexual and gender-based violence.

"Undoubted advantage is primary and secondary prevention when the medical examination succeeds in clarifying the consequences of doing and repeating mutilations. The medical examination can activate effective treatments in a timely manner, avoiding additional expenses in emergency situations of life."

Programme Implementer, Italy

"The advantages are that women and girls are made to be aware about the types and practices of $F G M / C$ and other preventive strategies on reproductive health including the protection of women against sexual and gender-based violence."

Programme Implementer, DRC

Another advantage of physical examination was the assistance it offers in the documentation of asylum claims for parents and girls trying to avoid being cut while living in communities where FGM/C prevalence is high. In some of the western countries, women seeking asylum due to $\mathrm{FGM} / \mathrm{C}$ are required to prove their status. Physical examination in this case was reported to be more reliable as it provided supporting documents that were used in the application for asylum.

"Beyond empowering and educating women about their own bodies, this [physical examination] can serve as supporting documentation for asylum claims, which is important in my work."

Healthcare Provider, USA

\section{Challenges/limitations of physical examinations}

Participants noted that in contexts where $\mathrm{FGM} / \mathrm{C}$ is illegal and reporting by healthcare providers is mandatory, FGM/C survivors may avoid routine visits to health facilities. Punitive anti-FGM/C laws cause fear among FGM/C survivors and antagonise the relationship between healthcare providers and patients. In such an environment, FGM/C survivors are likely to develop poor healthseeking behaviours, especially for cases that involve physical examination of their genitals.

"Women first have to go to health facilities. In the context of strong anti-FGM laws, women can avoid medical consultations for fear of denunciations. It is up to healthcare providers to build trust with women." 
The other challenge was the lack of protocol on how physical examinations related to FGM/C are to be conducted. Study participants noted that there were no guidelines on how to conduct physical exams, especially when dealing with women likely to have undergone FGM/C. They highlighted that healthcare providers are rarely trained on how to handle women and children who may have undergone FGM/C. Physical examination is an invasive procedure and is likely to be traumatic to women and girls if handled unprofessionally-especially in cases where it is done without the consent of the patient.

"The healthcare providers might physically examine their patients without express consent and permission [to determine FGM/C status] of the survivors which is a gross violation of their fundamental human rights."

Programme Implementer, Nigeria

"The challenges are in the training of healthcare providers: the examination must be done in an adequate way, preceded by a discussion which explains what they will do, look at the drawings [types of $F G M / C$ ] and in a non-judgemental way, not stigmatise (especially in Europe). The provider must also be able to recognise the different types of cut, possible complications, and know where to turn if necessary. Special attention should be paid to children because an examination of the genitals may be perceived as invasive or traumatic in children if it is not done adequately by explaining to the child what is going to happen, taking time, and avoiding many people being in the room during the exam."

Researcher and Healthcare Provider, Belgium

Limited capability of the healthcare system to document and report FGM/C was reported as a challenge related to physical examination. Participants highlighted the unavailability of tools in health facilities that would assist in recording and documenting FGM/C cases, which is critical in tracking its epidemiology. The healthcare personnel also noted a lack of training on the documentation of $\mathrm{FGM} / \mathrm{C}$, and in most cases there are no clear policy guidelines to support data collection related to FGM/C. Additionally, in most of the health facilities, there are few healthcare providers serving a huge population and therefore the excess workload makes it impossible to conduct physical examinations and document findings.

"The challenge is lack of awareness of FGM.... It needs to be taught in medical schools so that they [healthcare providers] can recognise it in clinical exams and record in medical records so that epidemiological figures are a reality."

Healthcare Provider, France

"They need proper training, and a good system for documentation-this should explain anatomical extent rather than ticking a type. It might be a challenge, though, in many countries where healthcare providers have reporting duties, it may not be feasible."

Researcher, Norway

Study participants mentioned the lack of knowledge about FGM/C among healthcare providers as a challenge in conducting physical examinations. They noted that a majority of healthcare providers, especially in the West, have limited experience with and knowledge about FGM/C. Such providers lack the capacity to conduct physical examinations in a professional manner when dealing with women or girls likely to have undergone FGM/C. Specific training needs that were highlighted included the identification of the different types of FGM/C, the varying morphology of the woman's genitalia, and challenges in diagnosis and management of FGM/C-related complications.

"In Italy, $80 \%$ of medical staff are not informed about FGM, do not know how to recognise them, do not know how to intervene, do not respect women and instead show them as 'objects of study' to colleagues, without even offering them appropriate care."

Programme Implementer, Italy 
"Physical examination will not show any alterations when pricking has been done...very few clinicians are aware of what the range is of 'normal' genitalia in adult women, and even fewer know the physical modifications that occur across growth and development in childhood. The lack of visible appearance of a clitoris has been mistaken for clitoridectomy when in reality the clitoris had not yet grown and emerged."

Researcher, USA

'Studies on healthcare providers' awareness, knowledge, and attitudes regarding FGM/C have shown lack of awareness of the prevalence, diagnosis, and management of FGM/C. They may be unfamiliar with cultural issues explaining the persistence of the practice or could find it difficult or uncomfortable to ask about it because of the fear of embarrassing or causing distress to their patient."

Researcher and Healthcare Provider, Netherlands

Participants noted that physical examination could lead to a resurgence of trauma for FGM/C survivors. This is likely to happen when the examination is performed by inexperienced providers sometimes without the consent of the patient.

"This can be an area of potential re-traumatisation if physicians are not thoughtful about describing their procedures and gathering information from their patients prior to performing physical exams."

Healthcare Provider, USA

"The examination can also cause the FGM trauma to arise again as it can be somehow similar to the FGM act."

Researcher, Finland

Participants were of the view that physical examination could amount to ethnic profiling if the examination was not done with every woman/girl in a given setting. It was noted that immigrant women from countries that have traditionally practised $F G M / C$ are in most cases specifically targeted for physical examinations with the aim of assessing their FGM/C status. When FGM/C is less common, physical exams were considered discriminatory because individuals are profiled based on their background information such as ethnicity and country of origin.

"Unless this is applied to everyone, whatever their background, it will rightly be perceived as racist. Women or girls who have been cut may well not turn up for the examination. It may mean that cutting is delayed to an older age rather than being prevented."

Researcher, UK

"If a woman is undergoing a regular or necessary gynaecological exam, it makes sense to see if there is cutting of some kind, especially if she has symptoms that could plausibly be related. The suggestion to do an exam on certain women (based on ethnic profiling) specifically to look for cutting for the purposes of reporting...would be much harder to justify."

Researcher, USA

Physical examination was noted to be a source of shame and discomfort among FGM/C survivors. In contexts where $\mathrm{FGM} / \mathrm{C}$ is frowned upon and providers have less experience with $\mathrm{FGM} / \mathrm{C}$, women who have undergone $\mathrm{FGM} / \mathrm{C}$ may feel uncomfortable undergoing the physical exam as they stand to be judged as having done something wrong that does not fit with the society's expectations. The level of shame and discomfort was reported to be worse in circumstances where the examination was conducted by a male provider.

"Migrant women are often afraid and ashamed to be visited. If the medical staff is male, women will feel humiliated."

Programme Implementer, Italy

Participants spoke about the ethical challenges that physical examinations pose. These ethical challenges included enforcement of laws requiring healthcare providers to report FGM/C cases 
while at the same time attending to the needs of the patient (common in Europe and the United States). This was noted to potentially do more harm to children if reporting FGM/C led to the child being separated from her parents. Other ethical challenges included making physical examination a requirement before access to other benefits; the misuse of authority by conducting physical exams without consent from women; intrusion of a woman's privacy that causes trauma, especially for underage girls; and challenges with consent and implementation of physical exams outside a health facility setting.

"The ethical challenge is that physical examination should not be a constraint for patients to obtain certain benefits (asylum law, naturalisation, etc.), but must be integrated into the follow-up care of patients in general rules without stigmatisation."

Healthcare Provider, France

"Informed consent, privacy, confidentiality, and do no harm...it could be doing harm to the child if uncovering a positive FGM/C status might lead to separating the girl from otherwise caring parents."

Researcher, Norway

"It is very intrusive. Why should women or girls undergo unnecessary pelvic exams for what-documentation purposes?...Unless they are treating women for gynaecological check-ups or ailments or during pregnancy I see no reason why healthcare providers should be examining anyone to report FGM/C status. You may find instances where girls are subjected to these physical exams without their consent which can also be harmful."

Researcher, Kenya

\section{Feasibility of conducting physical examinations on women/girls to collect data on FGM/C}

Given the challenges of physical examinations, we asked professionals working on FGM/C about their views on the feasibility of conducting physical examinations on women and girls with the aim of documenting FGM/C. The general view was that though expensive, it was possible to conduct a successful physical examination to assess $\mathrm{FGM} / \mathrm{C}$ but there were certain requirements that need to be fulfilled. These conditions included:

- Conducting the exercise in a health facility and ensuring consent from women and girls: "Most accurate when women come in pregnant or in labor, as most of them will at some point have children. For children, feasible only if routine genital examinations (for girls, by female providers) were instituted as a normal part of the annual physical for all children, which parents and children would come to expect."

Healthcare Provider, USA

"It is appropriate in the given clinic. The exam would need to make sense for the reason that patient is visiting a doctor. If it's outside the scope of the visit, it's not reasonable to ask to do a genital exam based on assumption."

Researcher and Healthcare Provider, Australia

- Integrating the exam with other health services provided at the health facility such as antenatal, postnatal clinics, immunisation clinics, and general gynaecological examinations:

"The easiest is for naturally occurring data to be collected at antenatal clinics, etc. However, the sample will be skewed in favour of those who are most informed about the value of antenatal care and not necessarily representative of the whole cohort."

Researcher, UK

- Conducting extensive training among healthcare providers about FGM/C for correct identification and classification of the various forms of $\mathrm{FGM} / \mathrm{C}$;

"You have to have good clinical observation rounds. You will need to train health workers well to recognise the difference between the normal anatomy of the woman's genitalia and the different types of cuts." 
"I would assume it would be an expensive exercise, you have to train the healthcare providers first (make sure they understand and are aware of FGM/C). The success also depends on whether women visit healthcare facilities. If you are in region where health facilities are not easily accessible or the rate of skilled births in health facilities is low, how will you make sure these providers can actually physically examine a good number of women-are they going to do outreach in the community to reach those at home or will the sample be biased to those who only visited the health facility?"

Researcher, Kenya

- Guaranteeing free pre- and post-medical examination care for women.

"It would not have to be very difficult in a clinical setting where women and girls come anyway. While this can pose a bias, it is most likely the best method. As long as it does not challenge reporting duties. And it should be used or combined with an opportunity for women for a free health check and provision of care."

Researcher, Norway

"You need well-trained doctors both to examine as well as for the aftercare. What do you offer the women who request help afterwards, both physically as well as mentally?"

Policymaker, Netherlands

\section{Advantages and challenges of women and girls self-reporting on FGM/C}

Self-reporting has been one of the most common methods of collecting data on FGM/C. National surveys such as the multi-country Demographic and Health Surveys (DHS) have consistently relied on self-reported data on FGM/C status to estimate FGM/C prevalence and its correlates. Given the popularity of self-reporting as a method of data collection for $F G M / C$, we sought to find out what professionals working in the area of $F G M / C$ think about this approach.

\section{Advantages/strengths of self-reporting}

Compared to physical examination, self-reporting was considered less intrusive. Self-reporting was noted to allow women and girls to describe their experiences with FGM/C to the best of their knowledge. Furthermore, risks associated with physical examination such as women feeling ashamed or uncomfortable were drastically reduced when self-reporting was used.

"Self-report is less invasive; it allows for better description of the [FGM/C] experience by women."

Researcher, Nigeria

"No risk relating to negative consequences of exams-shame, feeling of violation if exam is unwanted or medical professional isn't properly trained."

$$
\text { Programme Implementer, Belgium }
$$

Participants noted that self-reporting ensured that more women were reached and wide-ranging information captured within a short period of time. Self-reporting was used in surveys because it was a faster approach and made it easier to acquire data on sensitive issues such as $\mathrm{FGM} / \mathrm{C}$. Since the approach was non-invasive, chances of refusal by interviewees to participate in the study were noted to be lower and therefore studies were more likely to achieve the desired sample size. The approach was also advantageous because it does not require the interviewer to have a medical background.

"Able to carry out a household survey and interview a genuinely randomly selected sample. Interviewees do not need to have medical knowledge." 
"In my country's context this is more workable. Possible to collect data relatively easily and able to obtain subjects' consent. From the experience we learned that women or girls do not hide their circumcision status."

Researcher, Ethiopia

Self-reporting was noted to make it possible to document forms of FGM/C that are not easy to see physically. Some forms of FGM/C especially those described under Type I and IV may not leave a scar after healing and therefore a self-description of the events experienced by the woman was considered a more appropriate approach in documenting these types of FGM/C.

"It is possible to find the women with FGM Type I and IV. That wouldn't be found in the genital examination."

Researcher, Finland

Participants believed that self-reporting respects women's autonomy. Self-reporting was noted to give women an opportunity to express themselves and make the decision on whether they would want to participate or not and what they would like to respond to. It was also observed that women have a better understanding of their bodies and therefore are better placed to describe their experiences and the anatomy of their bodies.

"Excellent opportunity to allow women to describe their genital anatomy and experience of any type of cutting themselves."

Health Provider, USA

"They are the ones who are most familiar with their bodies and what they have undergone. So, they should be the ones to report on their own FGM/C status."

Researcher, Kenya

\section{Challenges and limitations of self-reporting}

The first challenge with self-reporting as a method of data collection was the definition of what FGM/C entails. Participants reported that even after an interviewer had described what FGM/C comprised, women may still not be aware of the different modifications because of a lack of a reference point. Women may also hold a completely different definition of what qualifies as FGM/C and what does not.

"Disadvantage is that you cannot be sure that what they are reporting is correct as women often get their 'mutilation' wrong...May get their type wrong or think that they are really mutilated even though their clitoris is still intact."

Researcher, Belgium

"Women may also define FGM differently, as in some cultures "Sunna," for example, can be seen as something other than FGM."

Researcher, Finland

"The researchers need to probe to ensure that there is a common understanding of the practice. For example, in some communities in Imo State in Nigeria where the people massage the baby's clitoris with ointments and hot water to desensitise it, they do not consider it FGM or FGC since flesh is not removed. So, if the question is not put in the proper context, they will not see it as a form of Type IV."

Programme Implementer, Nigeria

Closely related to the definition of FGM/C is the challenge relating to the description of the types of FGM/C. Participants noted that women may have a limited understanding of the anatomy of the female genitalia or may struggle to relate the morphology of her genitals with the professional language used to describe the different types of $F G M / C$.

"They might not have a clear or basic understanding of normal anatomy and language to describe genitalia limiting their ability to accurately describe their own altered anatomy."

Healthcare Provider, USA 
"Many women know they have been cut, but not many women (cut or uncut) are able to fully label and appreciate their own anatomy. I have found that countless women are convinced they have had their clitoris cut, only to do an exam and see only removal of the hood or a piece of labia. It's very common for patients to not fully understand what has been cut and to never have looked, assuming their anatomy is now drastically altered."

Researcher and Healthcare Provider, Australia

Self-reporting was noted to be negatively affected by the inability of respondents to recall FGM/C events in detail. Participants explained that women may find it difficult to recall events if $\mathrm{FGM} / \mathrm{C}$ was conducted at a younger age. This limitation was reported to be more pronounced when mild forms of FGM/C are performed during infancy leaving no visible alterations to the genitalia after healing.

"They may have been cut at a very young age and therefore don't remember what happened to them. They may have difficulty relating the state of their genitalia to official definitions."

Researcher, UK

"There might be under-reporting in communities where girls are cut in infancy and most don't recognise what a healthy, uncut vulva looks like."

Programme Implementer, Nigeria

Participants also felt that the accuracy of self-reported status is adversely affected by the context in which the interview was conducted. For example, in cases where FGM/C is illegal, women are likely to not tell the truth for fear of prosecution. Women's responses may also be affected by stigma especially if the woman lives in a setting where FGM/C has been condemned and majority of women do not practise it.

"It all depends on the context-if you interview women from an area where anti-FGM/C campaigns have taken place or it is well known that FGM/C is illegal, perhaps the women will have social desirability bias and report what they think the researchers want to hear (over-report "good" behaviour or under-report "bad" behaviour)."

Researcher, Kenya

The accuracy of self-reporting could also be compromised by a reluctance to talk about FGM/C. Opening up about intimate issues was reported to be a challenge as women may feel ashamed or reluctant to discuss issues relating to their genitals. They may also fear the consequences of talking about issues related to their reproductive health such as $\mathrm{FGM} / \mathrm{C}$ if by doing so they go against the established norms in their communities.

"Women migrants, refugees, asylum-seekers are afraid and ashamed to talk about the mutilations they have suffered. If they do, it is because pain and fear of the consequences are stronger than shame.... They are ashamed to talk about intimate aspects."

Programme Implementer, Italy

"Culture of silence. Tradition forbids women talking about their reproductive health."

Programme Implementer, Nigeria

\section{Validity of responses from parents/guardians reporting their daughter's FGM/C status}

Similar to women self-reporting on their FGM/C status, parents/guardians have been asked to report on their daughters' FGM/C status in national surveys such as the DHS. When asked whether parents' or guardians' reports on their daughter's FGM/C status were valid, participants noted that the validity of their responses was dependent on a number of factors:

First is the existence of laws prohibiting FGM/C. Criminalisation of FGM/C was meant to deter people from engaging in the practise, and thus affects people's honesty about the practice. 
Participants noted that parents may honestly report about their own FGM/C status especially if it happened many years ago because they cannot be prosecuted. However, reporting about FGM/C conducted on a minor in their custody could lead to prosecution and therefore in such situations the validity of parents' or guardians' responses was questionable as they are likely to under-report.

"Depending on relation between reporter and parent, increased criminalisation makes it harder for parents to honestly answer this question."

Policymaker, Netherlands

"It depends on how it is done, who asks the questions and the legal status. In Belgium, for example, where FGM/C is illegal it is unlikely that a parent would admit to having had their daughter undergo FGM/C. I have also noted that some fathers, especially if the daughter doesn't live with them, may not know if the daughter has had it. Or they may say they don't know in order to not have to confront it."

Programme Implementer, Belgium

"I think it is definitely less accurate, particularly where FGM/C is illegal and the parents/guardians are liable to legal action. Stigma (whether linked to being cut or uncut) might also lead to incorrect reporting."

Researcher, Norway

The prevalence of FGM/C in the area where parents reside was reported to affect the validity of their responses on the daughter's FGM/C status. In contexts where FGM/C prevalence is high and the practise considered a norm, parents or guardians may be pressured to report that their daughters have been cut even though they may not have been cut, just to conform with society's expectations and to avoid being stigmatised. The reverse was noted for contexts where FGM/C prevalence is low as parents are likely to feel pressured to deny FGM/C among their daughters because of stigma.

"Women don't talk about their private parts in public and because of societal norms in such community... if a woman is reported as not having been subjected to FGM in a community that expects every woman to be cut, the woman might be banished or sanctioned by the community."

$$
\text { Programme Implementer, Nigeria }
$$

"Some may not tell the truth. Because of stigma in the west, immigrant women may deny they were cut or state they were cut even if they were not, if it gains them an advantage in asylum proceedings or saves face within their own FGM-practising community."

Healthcare Provider, USA

Participants noted that ongoing anti-FGM/C interventions in the community can also affect the validity of parents' responses. They explained that in settings where anti-FGM/C interventions have been implemented for a long period of time, and community members have been educated about the negative health consequences of FGM/C, parents' responses on their daughters' $F G M / C$ status may be biased. This is because parents may not want to be seen condoning a practice that has been condemned during educational campaigns.

"All depends on the context. Parents/guardians would probably report accurately on their daughter's status as they would have been involved in the planning of the procedure. However, they may not want to report accurately if they know FGM/C is illegal or if there are active campaigns in their community to end the practice (Social desirability bias). Assurances of privacy and confidentiality need to be given so they feel comfortable in disclosing their daughter's true status."

Researcher, Kenya

Whether a parent was a natural or foster parent was considered to have an effect on the validity of parents'/guardians' responses. For example, participants considered foster parents to be more likely tell the truth about the daughter's FGM/C status than natural parents. This situation was 
reportedly common in western countries where foster parents take custody of the girl after FGM/C has already happened.

"I do not think that real parents' responses will be valid. It is criminal in Canada so parents would not declare it in my opinion, unless it is a foster family."

$$
\text { Healthcare Provider, Canada }
$$

Whether the person interviewing parents/guardians was considered an "insider" or "outsider" was also noted to likely affect the validity of parents' responses on the daughter's FGM/C status. Participants believed that insiders interviewing parents/guardians are likely to get valid responses about the daughter's FGM/C status as compared to outsiders. Because of the sensitivity of matters related to $\mathrm{FGM} / \mathrm{C}$, parents and guardians would be uncomfortable sharing information concerning their daughter with someone they considered an outsider.

"I doubt most parents would admit to cutting their child in a Western culture, given the negative connotations around it now. Possibly this would be more effective in the communities where it is practised, but only if these questions were asked by members of that community. I believe the taboo/negative views of FGM are starting to leak into communities that practise it, meaning they would be less likely to disclose to an outsider."

Researcher and Healthcare Provider, Australia

\section{Preferred approaches in measuring FGM/C prevalence}

Opinions on which approaches were preferred in measuring $\mathrm{FGM} / \mathrm{C}$ prevalence were varied. Nonetheless, majority of study participants preferred combining multiple approaches in collecting data in order to boost the accuracy of FGM/C prevalence measurements. The most preferred combination was the use of physical examinations by healthcare providers and self-reported surveys of women in the community.

"Using a variety of measurements for triangulation,...perhaps combining self-reporting using skilful interviewers comparing data sets over time, focusing on young women 15-24 in particular, with $\mathrm{MCH}$ [maternal and child health] data from local health facilities. In addition, using FGDs [focus group discussions] to explore changes in attitudes."

Researcher, UK

"Continue to have FGM modules in DHS studies with the indirect method because they allow for large and national samples...but cross DHS results with routine clinical consultation records in prenatal consultations for women adults and...maternal and child protection consultations for children (doing it in an integrated way is much less invasive and it gets into the routine)."

Researcher and Healthcare Provider, Belgium

"Self-reporting for large-scale population-based studies combined with smaller scale physical examination to get more insight to anatomical extent and health complications."

Researcher, Norway

Those who preferred physical examination of women in a health facility setting argued that physical examination with a trained healthcare provider was a more reliable method for verifying whether a woman/girl has been cut. They emphasised the need to integrate this approach into other routine examinations happening at the facility especially during ante- and post-natal clinics, family planning clinics, and clinics for children younger than five years.

"Doctors need to include this during medical appointments or interviews and follow it up with clinical examinations as proof."

Healthcare Provider, France

"Physical examination with the proper ethical clearance and permissions is a better verifiable method." 
"Physical examination at routine clinics including under-5 infant clinic, family planning clinics, and maternity clinics."

Programme Implementer, Nigeria

Those in support of the use of self-reports noted that this was the most viable way to measure prevalence in large-scale surveys. They noted that compared to physical examination, selfreported surveys are easier to implement and do not require a healthcare professional during data collection.

"Self-report and report on daughter's status are useful in prevalence studies. In order to glean prevalence from medical records, providers would need extensive training, and would need to record this consistently. This does not happen in most countries."

Researcher, USA

\section{Views about WHO classification of the different types of FGM/C}

Standardised classification can ensure comparability of datasets. A standardised classification is equally beneficial in research on the consequences of the different types of FGM/C, estimation of FGM/C prevalence and trends, accurate gynaecological examinations and subsequent management of complications related to $\mathrm{FGM} / \mathrm{C}$, and can assist in court proceedings (OHCHR et al. 2008). Classification of the various forms of FGM/C into four distinct groups by WHO has for a period generated debate on the relevance and applicability of the classifications. We sought to find out from professionals their thoughts and experiences using the $2008 \mathrm{WHO}$ classifications of $\mathrm{FGM} / \mathrm{C}$.

Participants raised their concerns about challenges with the definition of FGM/C Type IV. They were of the view that Type IV lumps together all the undefined forms of interference to the female genitalia making it difficult to categorise and measure prevalence. The lack of specificity opens room for confusion where people are left on their own to determine whether the interference with the female genitalia qualifies as FGM/C or not.

"Type IV is a catch-all category. I think pricking that does not remove health tissue and permanently alter the genitalia should be a single category, and not put into a catch-all category."

Researcher, USA

"The Type IV is not well defined and as such poses a challenge in determining the prevalence. There are lots of practices involving the female genitalia that does not disfigure the genitals. So, measuring that may not be achieved."

Researcher, Nigeria

Healthcare providers highlighted the difficulty in identifying types of FGM/C. This was because there are no clear differences between Types I and II, and it was not always possible to see the alterations by observation to be classified under Type IV.

"The usefulness of the classification system is limited and confusing and often doesn't help point a practitioner to necessary intervention. I think most doctors would be able to recognise Type III, but the nuances between the other types would be lost because of how the current system is set up."

Researcher and Healthcare Provider, Australia

"It's very difficult to measure because sometimes there are invisible traces after healing of an incision on the clitoral hood. In addition, it covers several unclassified types such as cautery, a cut inside the vagina-we could try to do this with the indirect method with a sufficiently broad question: during your childhood do you remember that you were hurt ..... with products that burned or stung, .... and the person could describe this? It will be difficult to measure prevalence, but it may be a starting point for further qualitative research... So, there is still a whole field to explore in Type IV." 
"I have never personally seen a Type IV (I am a gynaecologist in New York) so would have to deduct it from a patient's story and be trained to see what it looks like years later, if there still are scars. Seems a bit like a category where you dump everything that you don't exactly know what happened."

Healthcare Provider, USA

Participants were of the view that the classifications by WHO need improvement and do not provide enough guidance for healthcare workers.

"Beside the fact that the WHO classification does not encompass/reflect all variations in the anatomical presentation of FGM/C nor the severity of the cutting, or the fact that many women and healthcare providers are not familiar with the classification, I think the WHO classification is in dire need of improvement, and Type IV in particular is very problematicnot only with regard to measuring prevalence. Piercing and nicking for example are both Type IV, but it is highly unlikely to prosecute someone for performing piercing. Also, excluding female genital cosmetic surgeries from the classification while including nicking sounds more like double standard."

Researcher, Norway

"The WHO classification is a poor representation of what is necessary to know during an exam. This classification needs to be restructured from a healthcare perspective... Examination and classification are incredibly difficult. Additionally, a majority of healthcare providers have never seen FGM/C and certainly aren't familiar with the classification of $F G M / C$. This lack of knowledge and education makes exams uncomfortable and unnecessary for patients-they end up becoming a teacher versus having come to a doctor expecting answers."

Researcher and Healthcare Provider, Australia

\section{Feasibility of using images in identifying and classifying various types of $\mathrm{FGM} / \mathrm{C}$}

Given the debate around classification of the various types of $F G M / C$, we investigated study participants' views and experiences on the use of images/pictorial presentations to help identify and classify FGM/C. Apart from ethical challenges that may confront those intending to use images in the identification and classification of $\mathrm{FGM} / \mathrm{C}$, pictorial presentations were considered to be helpful in improving the accuracy in the identification and classification of $F G M / C$. The following are the two main areas that could benefit from the use of images:

Participants explained that anatomical drawings can be very helpful in improving the documentation of the extent of FGM/C and can educate women. The images were deemed helpful both in scenarios where the healthcare provider was expected to observe and record, and in situations where a woman was shown the images to identify the type of FGM/C that she had experienced.

"The images are incredibly helpful. I am a visual learner, so for me that's the resource I started using. While the WHO images are fairly accurate, there would be easier ways to guide unfamiliar doctors through the classification system. Currently, it requires a lot of back and forth scrolling to understand where your patient is (if you aren't familiar with the classification system)."

Researcher and Healthcare Provider, Australia

"I use them in consultation, I show the woman the different types drawn (no photo, it bothers women less) and I invite the woman to put her finger on the drawing that resembles her anatomy. If she doesn't know, I say that it doesn't matter that I will look at the exam and that I will explain to her after what I saw, I can also invite her to look with a mirror (but I don't force). Some Somali women who have been de-infibulated can even tell their 
trajectory with the drawings: small infibulated, then open during childbirth, then closed after."

Researcher and Healthcare Provider, Belgium

"Images are necessary to train doctors but also to educate women and help them understand that every vulva is different and what an anatomically 'correct' vulva looks like."

Policymaker, Netherlands

But participants cautioned that actual photos, while more useful for providers, can raise ethical issues. Drawings were seen as more acceptable to a broader audience.

"It is okay for training sessions to aid understanding. However, when working with communities it is important to desensitise the participants before showing the images (not the graphic ones). One should also find out if it is considered appropriate to show such images in the community."

Programme Implementer, Nigeria

"In trainings, it is necessary to use images for healthcare professionals, including real photos. I do not think images, especially photos, need to be used all the time in trainings and awareness-raising. The use of photos has been said to be useful to raise awareness of the problem, for example among men, but it raises many ethical issues on how you show images of (generally black) women's bodies. The photos used also typically show the 'worst' types and the complications. I think that the use of these kind of photos can have a negative impact on women and girls affected by $F G M / C$ and their self-esteem and perception of their bodies."

Programme Implementer, Belgium

"Not good in a country where seeing and talking of private parts are taboo like my country." Researcher, Ethiopia

"I am on the fence-if they use illustrations/drawing to help classify the types then I am okay. But if you are using actual pictures of women's genitalia then I am against it, unless there are assurance that the woman has given consent for the images to be used."

Researcher, Kenya

\section{Discussion}

This study set out to investigate the views of professionals involved in FGM/C work about the advantages and challenges of physical examination and self-reporting in collecting data on $F G M / C$, the validity of responses from parents or guardians reporting on their daughter's FGM/C status, preferred approaches in measuring FGM/C prevalence, and the WHO classification of the different types of FGM/C. Study findings on the advantages and disadvantages of physical examinations showed that the approach has strengths but at same time is faced by numerous challenges. Some of the strengths of physical exams include: opportunity for identification and treatment of health complications related to $\mathrm{FGM} / \mathrm{C}$; greater reliability of status data compared to self-reports; opportune moment for sensitisation and prevention of $\mathrm{FGM} / \mathrm{C}$; and critical documentation of asylum claims based on FGM/C. Key challenges in conducting physical exams include the high possibility of FGM/C survivors avoiding routine visits to health facilities in contexts where healthcare providers are required to report cases to authorities; lack of protocol on how physical examination is to be conducted; limited capability of the healthcare system to document and report $\mathrm{FGM} / \mathrm{C}$ cases; inadequate knowledge about FGM/C among healthcare providers; resurgence of trauma for FGM/C survivors; possibility of ethnic/racial profiling if the exercise does not apply to the general population; and experience of shame and discomfort among FGM/C survivors.

These findings are not unique as research on physical/clinical examinations of women with the aim of determining their FGM/C status has shown that even though clinical exams are regarded as the 
gold standard (Elmusharaf, Elhadi, and Almroth 2006; Klouman, Manongi, and Klepp 2005) the approach has numerous limitations (Adinma 1997; Morison et al. 2001; Odujinrin et al. 1989; Snow et al. 2002; Toubia 1994). The limitations stem from the complexity involved in conducting the exercise and the vast amount of resources required to make it a success (Huntington et al. 1996; Jones et al. 1999). Our study findings on the feasibility of conducting physical exams with the aim of documenting FGM/C indicated that though expensive, it is possible to conduct a successful exercise, but there are certain conditionalities that needed to be fulfilled. These conditions included: conducting the exercise in a health facility and ensuring consent from women and girls; integrating the exercise with other health services provided at the health facility such as ante- and post-natal clinics, immunisation sessions, and general gynaecological examinations; conducting extensive training among healthcare providers about $\mathrm{FGM} / \mathrm{C}$ for correct identification and classification of the various forms of $\mathrm{FGM} / \mathrm{C}$; and guaranteeing free pre- and post-medical examination care for women undergoing the exercise.

Compared to physical examination, self-reporting of FGM/C status was considered less intrusive; had the advantage of covering a larger population and capturing wide-ranging information within a short period of time; made it possible to document forms of FGM/C that cannot be physically seen; and was considered to respect women's autonomy. Similar to physical examinations, self-reporting was associated with various limitations such as difficulty in reconciling the interviewer's and interviewee's definition of FGM/C and the description of the various types of FGM/C; the negative influence of recall bias; the adverse effects of context especially in settings where FGM/C is illegal; and reluctance of women to talk about intimate topics such as FGM/C.

Research on the accuracy of women's self-reporting on their FGM/C status, comparing women's self-reports with subsequent clinical examinations has shown some variations in the accuracy. For example, a study conducted in Egypt in 1996 found a high agreement (92\%) between self-reported FGM/C status and gynaecological examination (Huntington et al. 1996). Another study conducted in the Gambia in 1999 exhibited even higher agreement (97\%) between the self-reported FGM/C status and gynaecological assessments (Morison et al. 2001). In Nigeria, two studies have assessed the accuracy of self-reports in a setting where $\mathrm{FGM} / \mathrm{C}$ prevalence is not near universal (Larsen and Okonofua 2002; Snow et al. 2002). One of the studies found a high level of agreement between self-reports and physical examinations (92\%) (Larsen and Okonofua 2002). Notably, 17\% of women interviewed reported that they did not know their FGM/C status, $9 \%$ of women who were confirmed to be uncut in an exam had self-reported that they were cut, and $6 \%$ of women were confirmed as cut through a gynaecological exam but had reported that they were uncut (Larsen and Okonofua 2002). The other study conducted by Snow and colleagues (2002) exhibited a comparatively lower level of agreement at $79 \%$ between clinical examinations and self-reports. Additionally, a considerable proportion of women (14\%) reported that they did not know their FGM/C status (Snow et al. 2002). Given the noted challenges of self-reporting and the highlighted variations in the accuracy of self-reporting in the literature, there is need for caution when interpreting data on FGM/C generated through self-reports (Snow et al. 2002). Specifically, the influence of context cannot be ignored. For example, the study in Egypt showed that self-reporting was accurate in $94 \%$ of cases-a context where the overall prevalence was $93 \%$ (Huntington et al. 1996), while a study in Nigeria found self-reporting accurate in $57 \%$ of cases-a context where the national prevalence was 48\% (Adinma 1997).

Closely related to self-reporting by women and girls about their FGM/C status is the issue about the validity of parents' or guardians' responses on their daughters FGM/C status. Our findings have shown that the validity of parents' or guardians' responses may be dependent on a number of factors, key of which are: the existence of laws prohibiting $\mathrm{FGM} / \mathrm{C}$; the prevalence rate of $\mathrm{FGM} / \mathrm{C}$ in the area where parents reside; ongoing anti-FGM/C interventions in the community; whether the respondent is a natural or foster parent; and whether the person interviewing the parent or guardian 
is considered an "insider" or "outsider". Research has shown that parents or guardians may deliberately misreport because of social desirability issues or for fear of prosecution in contexts where FGM/C is illegal (Jackson et al. 2003). That said, evidence exists that self-reports are largely valid (Bjälkander et al. 2013). It is therefore useful to highlight that parents'/guardians' reports are still the most viable way of collecting data on girls younger than 15 years who are often not included as participants in large-scale surveys.

Findings on the preferred approaches in measuring FGM/C were varied. Nonetheless, a majority of participants preferred a mix of approaches, particularly combining physical examination and selfreporting. Those in favour of a mixed-methods approach were of the view that triangulation of approaches in collecting data was likely to boost the accuracy in measuring FGM/C prevalence. Physical examinations in health facilities were preferred because they were more reliable in verifying women's FGM/C status. Those in support of the use of self-reports noted that it was the most viable way to measure prevalence in large-scale surveys. The inference from these findings is that approaches used in collecting data on FGM/C vary, with each approach serving a particular purpose. Practitioners using approaches such physical examinations and self-reporting need be cognisant of their strengths and limitations. This will assist them in interpreting data and its usage in monitoring FGM/C abandonment.

Study findings on the WHO classification of the different types of FGM/C highlighted various challenges in its relevance and application. Professionals working in the area of FGM/C noted challenges with the definition of FGM/C Type IV and the difficulty in identifying forms of $F G M / C$ to be categorised under Type IV. They also highlighted how these classifications make it difficult to prosecute FGM/C cases due to the ambiguity and double standard in some of the classifications, especially Type IV. The limited understanding of the WHO classifications of FGM/C among healthcare providers was mentioned as a challenge in identifying and managing $F G M / C$ cases. Whereas the WHO classifications of the different types of $\mathrm{FGM} / \mathrm{C}$ has been an important milestone in the description and comparison of the practice, the different forms of FGM/C are complex (ShellDuncan and Hernlund 2006; Shell-Duncan and Hernlund 2000). Precise anatomical classification relies on examination of the genitalia by an experienced examiner on FGM/C matters. Even then, discrepancies have been noted in women's reports and anatomical examination findings (Elmusharaf, Elhadi, and Almroth 2006). Importantly, even when gynaecological examinations are conducted by an experienced examiner, challenges still exist regarding clearly fitting genital findings into a particular type (Creighton and Hodes 2016).

Given the challenges in identifying and classifying various forms of FGM/C, our study sought to find out the feasibility of using images in identifying and classifying numerous types of $F G M / C$. Abdulcadir and colleagies emphasised the importance of images/drawings in aiding identification, classification and management of FGM/C cases (Abdulcadir et al. 2018). Apart from ethical challenges that may confront people intending to use images in the identification and classification of $\mathrm{FGM} / \mathrm{C}$, pictorial presentations, especially in form of drawings, were considered to be helpful in improving accuracy in the identification and classification process. The two main areas that would benefit from the use of images/drawings included documentation of the extent of FGM/C and use of images in knowledge dissemination about FGM/C.

\section{Limitations}

Data were collected from professionals working on FGM/C who attended the G3 International Expert Meeting on FGM/C and those whose contacts were available. Even though efforts were made to get views and experiences from different stakeholders across the globe, findings from this study may not be generalisable. Responses from the various professionals represent personal views and experiences in their area of work and do not represent views and experiences of all 
professionals working in the specific sector. Nonetheless, the combination of responses from various professionals from different countries helped to triangulate more representative insights. Lastly, many of the interviewees were Westerners and may represent a viewpoint different from those of people coming from communities where FGM/C is largely practised.

\section{Conclusion}

The debate on the most suitable methods for measuring $\mathrm{FGM} / \mathrm{C}$ has been longstanding. In response, this study has documented some of the key measurement aspects surrounding datacollection approaches on $\mathrm{FGM} / \mathrm{C}$. The complexity of $\mathrm{FGM} / \mathrm{C}$ makes research on this topic challenging and more thought needs to be given to the methods used in collecting data on the practice.

These findings on the various approaches of data collection with reference to FGM/C point to the need for practitioners using these approaches to be cognisant of the specific strengths and limitations of each approach. Clinical examinations are more suitable in a health facility setting and when it is integrated within routine services provided by the facility. Self-reports on the other hand are largely appropriate when conducting community surveys. Whereas the WHO classifications of the different types of $F G M / C$ has been an important step in aiding measurement of $F G M / C$, professionals working on $\mathrm{FGM} / \mathrm{C}$ reported several challenges when using these classifications. The use of images/drawings can complement other methods and can be a useful teaching aid both for providers and women and girls.

\section{Implications for Programmes and Research}

Findings on the various approaches of data collection related to FGM/C point to the need for programme implementers and researchers utilising these approaches to be cognisant of the specific strengths and limitations of each approach. Data generated from physical examinations, women's self-reports, or parents' or guardians' reports on their daughters' FGM/C status should be interpreted with caution, bearing in mind the limitations of the specific approaches. As a response to the ongoing debate over which data-collection approach is suitable for FGM/C, this study has documented some of the key opportunities and challenges regarding collection of data on the practice. This report is therefore a unique resource for programme implementers, researchers, and other stakeholders interested in the data-collection discourse on sensitive issues such as $\mathrm{FGM} / \mathrm{C}$.

There is need for systematic research on the relevance and applicability of the WHO classifications of the different types of FGM/C. This will shed more light on the challenges encountered by various professionals using these standards and offer practical solutions surrounding the noted ambiguities in the classifications, especially Type IV. More research should also be conducted to provide clear guidelines on how images/drawings can be used in the identification, classification, and management of $\mathrm{FGM} / \mathrm{C}$ cases. 


\section{References}

Abdulcadir, J., S. Marras, L. Catania, O. Abdulcadir, and P. Petignat. 2018. "Defibulation: A visual reference and learning tool," The Journal of Sexual Medicine 15(4): 601-611. https://doi.org/10.1016/j.jsxm.2018.01.010

Adinma, J.I. 1997. "Current status of female circumcision among Nigerian Igbos," West African Journal of Medicine 16(4): 227-231.

Askew, I. 2005. "Methodological issues in measuring the impact of interventions against female genital cutting," Culture, Health \& Sexuality 7(5): 463-477. https://doi.org/10.1080/13691050410001701939

Bjälkander, O., D.S. Grant, V. Berggren, H. Bathija, and L. Almroth. 2013. "Female genital mutilation in Sierra Leone: Forms, reliability of reported status, and accuracy of related demographic and health survey questions," Obstetrics and Gynecology International 14. https://doi.org/10.1155/2013/680926

Creighton, S.M. and D. Hodes. 2016. "Female genital mutilation: What every paediatrician should know," Archives of Disease in Childhood 101(3): 267-271. https://doi.org/10.1136/archdischild-2014-307234

Elmusharaf, S., N. Elhadi, and L. Almroth. 2006. "Reliability of self-reported form of female genital mutilation and WHO classification: Cross sectional study," BMJ 333(7559): 124. https://doi.org/10.1136/bmj.38873.649074.55

Gibson, M.A., E. Gurmu, B. Cobo, M.M. Rueda, and I.M. Scott. 2018. "Indirect questioning method reveals hidden support for female genital cutting in South Central Ethiopia," PLOS ONE 13(5): e0193985. https://doi.org/10.1371/journal.pone.0193985

Huntington, D., L. Nawar, N.A. Tawab, and S. Hegazi. 1996. Clinic-Based Investigation of the Typology and Self-Reporting of FGM in Egypt. Cairo: Population Council, Egyptian Fertility Care Society, and Macro International.

Jackson, E.F., P. Akweongo, E. Sakeah, A. Hodgson, R. Asuru, and J.F. Phillips. 2003. "Inconsistent reporting of female genital cutting status in Northern Ghana: Explanatory factors and analytical consequences," Studies in Family Planning 34(3): 200-210. https://doi.org/10.1111/j.1728-4465.2003.00200.x

Jones, H., N. Diop, I. Askew, and I. Kaboré. 1999. "Female genital cutting practices in Burkina Faso and Mali and their negative health outcomes," Studies in Family Planning 30(3): 219-230. https://doi.org/10.1111/j.1728-4465.1999.00219.x

Klouman, E., R. Manongi, and K.-I. Klepp. 2005. "Self-reported and observed female genital cutting in rural Tanzania: Associated demographic factors, HIV and sexually transmitted infections," Tropical Medicine \& International Health 10(1): 105-115. https://doi.org/10.1111/j.1365-3156.2004.01350.x

Larsen, U., and F.E. Okonofua. 2002. "Female circumcision and obstetric complications," International Journal of Gynecology \& Obstetrics 77(3): 255-265. https://doi.org/10.1016/S0020-7292(02)00028-0

Morison, L., C. Scherf, G. Ekpo, K. Paine, B. West, R. Coleman, and G. Walraven. 2001. "The long-term reproductive health consequences of female genital cutting in rural Gambia: A community-based survey," Tropical Medicine \& International Health 6(8): 643-653. https://doi.org/10.1046/j.1365-3156.2001.00749.x

Odujinrin, O.M., C.O. Akitoye, and M.A. Oyediran. (1989). "A study on female circumcision in Nigeria," West African Journal of Medicine 8(3): 183-192.

OHCHR, UNAIDS, UNDP, UNECA, UNESCO, UNFPA, UNHRC, UNICEF, UNIFEM, \& WHO. 2008. Eliminating female genital mutilation: An interagency statement. Geneva: WHO.

Shell-Duncan, B., and Y. Hernlund. 2000. Female "Circumcision" in Africa: Culture, Controversy, and Change. Boulder, CO: Lynne Rienner Publishers. 
2006. "Are there 'stages of change' in the practice of female genital cutting? Qualitative research finding from Senegal and the Gambia," African Journal of Reproductive Health 10(2): 57-71.

Snow, R.C., T.E. Slanger, F.E. Okonofua, F. Oronsaye, and J. Wacker. 2002. "Female genital cutting in southern urban and peri-urban Nigeria: Self-reported validity, social determinants and secular decline," Tropical Medicine \& International Health 7(1): 91-100. https://doi.org/10.1046/j.1365-3156.2002.00829.x

Toubia, N. 1994. "Female genital mutilation and the responsibility of reproductive health professionals," International Journal of Gynecology and Obstetrics 46(2): 127-135.

WHO. 1998. Female Fenital Mutilation: An Overview. Geneva: World Health Organization. https://apps.who.int/iris/bitstream/handle/10665/42042/9241561912_eng.pdf.

2006. "Female genital mutilation and obstetric outcome: WHO collaborative prospective study in six African countries," The Lancet 367(9525): 1835-1841.

https://doi.org/10.1016/S0140-6736(06)68805-3 